\title{
Maxwell-Bloch Equations as Predator-Prey System
}

\author{
A. S. Hacinliyan ${ }^{1,2}$, E. E. Akkaya ${ }^{1}$, I. Kusbeyzi ${ }^{2,3}$ and O. O. Aybar ${ }^{2,3}$ \\ 1 Yeditepe University, Istanbul, Turkey \\ Department of Physics \\ (e-mail: erguneray.akkaya@std.yeditepe.edu.tr) \\ (e-mail: ahacinliyan@yeditepe.edu.tr) \\ 2 Yeditepe University, Istanbul, Turkey \\ Department of Information Systems and Technologies \\ 3 Gebze Institute of Technology, Kocaeli, Turkey \\ Departments of Mathematics \\ (e-mail: ikusbeyzi@yeditepe.edu.tr) \\ (e-mail: oaybar@yeditepe.edu.tr)
}

\begin{abstract}
Regions of the full parameter space for which chaotic behavior in laser models based on the Maxwell-Bloch equation occurs are studied[1]. The range in the parameter space have been charted, all possibilities for the value of the maximal Lyapunov exponent are shown to exist, positive maximal Lyapunov exponents characterizing chaotic behavior occurs for parameter values that correspond to the range of parameters for Helium-Neon lasers. The Maxwell-Bloch equations as given by [2] and [3] involve the coupling of the fundamental cavity mode, $E$ with the collective variables $P$ and $\Delta$, that represent the atomic polarization and the population inversion. They are represented by the following equations.
\end{abstract}

$$
\begin{gathered}
\dot{E}=-k E+g P \\
\dot{P}=-\gamma_{\perp} P+g E \Delta \\
\dot{\Delta}=-\gamma_{\|}\left(\Delta-\Delta_{o}\right)-4 g P E
\end{gathered}
$$

For the parameter values $k=\sigma, \gamma_{\perp}=g^{2} / k=1, g^{2} \Delta_{o} / k=r, \gamma_{\|}=b$, the system can be transformed into the Lorenz system about the equilibrium point $\Delta=\Delta_{o}$ by setting $x=E, y=g P / k, z=\Delta_{o}-\Delta$. The meaning of the parameters in the original equations as given by Arrechi[2], while $\sigma, r, b$ are the Lorenz parameters.

Maxwell-Bloch equations can be transformed into a system proposed by [4], that resembles the Lotka-Volterra problem with $I$ representing the intensity of the laser field and $N$ denoting population density, the dimensionless rate equations for I and $\mathrm{N}$ are obtained by assuming $I=E^{2}$ and the subsidary condition $g P=k E \Delta$. Starting with the first Maxwell-Bloch Equation multiplying both sides by $E$ gives $E \dot{E}=-k E^{2}+g P E, \dot{P}=-\gamma_{\perp} P+g E \Delta=-\gamma_{\perp} P+g^{2} P / k$ The subsidary condition enables one to solve for the polarization as $P=P_{o} \exp \left(-\left(\gamma_{\perp} P-g^{2} / k\right) t\right)$ and the third equation becomes $\dot{\Delta}=-\gamma_{\|}\left(\Delta-\Delta_{o}\right)-4 k E^{2} \Delta$. Finally equations for the intensity and population density are obtained by defining $\Delta=N, 2 k t=\tau$ and $\widetilde{I}=2 I \gamma$. They are:

$$
\begin{gathered}
\dot{\widetilde{I}}=\widetilde{I}(-1+N) \\
\dot{N}=\gamma(A-N-N \widetilde{I})
\end{gathered}
$$


For $A=0$, these equations resemble the Lotka-Volterra equations[4]. Thus the population fluctuations in the laser system emulate a predator prey system governed by the pumping and lasing actions. These equations possess two equilibrium points, $\widetilde{I}=0, N=A$ or $\widetilde{I}=\gamma(A-1), N=1$. The former equilibrium point when linearized has eigenvalues $A-1,-\gamma$ so that a bifurcation is not immediately expected. An integer ratio between $A-1$ and $\gamma$ can indicate resonances in the normal form expansion. The latter point when linearized has eigenvalues $\frac{-A \gamma \pm \gamma \sqrt{4-4 A+A^{2} \gamma}}{2}$ so that when $4-4 A+A^{2} \gamma$ changes sign, a transition of domain indicating a Hopf bifurcation can occur. The normal form expansion about this equilibrium point is not expected to yield a resonance condition[5].

We see that these type of lasers naturally exhibit damped intensity oscillations that satisfy a degenerate form of the Lotka-Volterra equations. Lotka-Volterra equations appear as an underlying problem and allow us to describe the bifurcation that has been also numerically verified in the original system.

Keywords: Maxwell-Bloch equations, Hopf Bifurcation, Chaotic behaviour, Optical Nonlinearity.

\section{References}

1.A. Dhooge, W. Govaerts, Yu.A. Kuznetsov, W. Mestrom, A.M. Riet and B. Sautois,. MATCONT and CL_MATCONT: Continuation toolboxes in matlab. Ghent and Utrecht Universities Preprint, 2006.

2.F. T. Arecchi. Chaos and Generalized Multistability in Quantum Optics. In A.F. Round, editor, Physica Scripta, 85-92, 1985.

3.H. Haken. Laser Light Dynamics, Volume 2, 1985.

4.Thomas Erneux and Gregory Kozyreff. Nearly Vertical Hopf Bifurcation for a Passively Q-Switched Microchip Laser. Journal of Statistical Physics, Vol. 101, 1-2, $543-552,2000$.

5.A. Hacinliyan, N. Z. Perdahçı, G. Şahin, H. A. Yıldırım. Transforming to Chaos by Normal Forms. CMDS 10, Shoresh, Israel, 15-20, 2003. 